\title{
Nonlinear dynamics and performance enhancement of asymmetric potential bistable energy harvesters
}

\author{
Wei Wang $\cdot$ Junyi Cao $\cdot$ Chris R. Bowen $\cdot$ Ying Zhang $\cdot$ Jing Lin
}

Abstract Bistable systems exhibiting complex dynamic behaviors have been viewed as efficient methods to overcome the issue of linear energy harvesters only performing well near their resonance frequencies. Moreover, performance enhancement strategies of bistable energy harvesters have been extensively discussed for perfectly symmetric potentials. Due to imperfects caused by non-uniform manufacturing of the harvester, eccentricity of the buckling or magnetic force, and uneven gravity, dynamic characteristics and performance enhancement of asymmetric potential energy harvesting remain an open issue. Therefore, this paper investigates the influence mechanism and performance enhancement method of cantilever-based bistable energy harvesting systems with asymmetric potentials. Bifurcation diagram is employed to discover the effect of asymmetric potentials on the output response of the dimensionless electromechanical equation. Based on the numerical results, a performance enhancement method is proposed by compensating the asymmetric potentials with a proper bias of the system for decreasing the negative impact of asymmetric potentials on bistable energy harvesting. The optimum bias angle is derived and numerical simulations under constant and sweep frequency excitations demonstrate that the performance of the asymmetric potential BEHs is enhanced in a certain bias angle range around the optimum value. Two bistable energy harvesters with different asymmetric potential functions are investigated in the experiments and results verify

W. Wang $\cdot$ J. Cao $(\bowtie) \cdot$ Y. Zhang $\cdot$ J. Lin

Key Laboratory of Education Ministry for Modern Design and Rotor-Bearing System, Xi'an Jiaotong University, Xi'an, 710049, China

e-mail:caojy@mail.xjtu.edu.cn.

C. R. Bowen

Materials and Structures Centre, Department of Mechanical Engineering, University of Bath, Bath, BA27AY, UK

e-mail: c.r.bowen@bath.ac.uk 
the effectiveness of the proposed method for improving the energy harvesting performance.

Keywords Energy harvesting $\cdot$ Bistable $\cdot$ Asymmetric potential $\cdot$ Nonlinear dynamics $\cdot$ Performance enhancement 


\section{Introduction}

Vibration energy harvesting technique has been viewed as a promising method to enable self-sufficient wireless sensors and other low-power consumption devices on account of the presence of vibration energy in numerous surroundings and engineering systems[1-3]. Among different vibrational energy conversion mechanisms, piezoelectric transduction based configurations[4, 5] have been the most favored due to the high energy density and easily miniaturized fabrication. In last decades, piezoelectric energy harvesters based on traditional linear resonance mechanism have been widely investigated. However, the main issue of linear harvesters is that they will become incapable or even failed when subject to ambient vibrations with a wide frequency range[6].

To overcome the issue, theoretical and experimental validation of the frequency bandwidth and performance enhancement of harvesters as a result of introducing nonlinearity[7,8] has received significant attentions. Monostable[9, 10], bistable[11-14], and tristable[15, 16] oscillators with different types of potential functions were widely investigated. Stanton et al.[17] obtained a monostable energy harvester (MEH) by tuning the magnetic interactions around the end mass and verified its capability to outperform linear harvester. Zhou et al.[18] investigated the multi-solution of a $\mathrm{MEH}$ and demonstrated that appropriate initial conditions can realize high-branch oscillation for generating more energy. Due to the broadband snap-through responses, bistable energy harvesters (BEH) resulting from nonlinear and mechanical mechanisms have been widely employed to improve the power generation in energy harvesting[2]. Erturk et al.[19] introduced a magnetoelastic structure with double well to energy harvesting and proved its superiority to linear case. Daqaq[20] studied the response of a bistable harvester to white Gaussian excitations and indicated the shape of potential energy function influences the output power significantly. Moreover, Li et al.[5] observed the 
coherence resonance of the bistable harvester subjected to Gauss white noise excitations which can be applied to optimize the efficiency. More recently, a variety of magnetic-coupled configurations with three or four stable potential wells have been reported by Zhou et al.[21, 22], Kim and Seok[15, 23, 24], Daqaq[25], and Qin et al.[26-28] for enhancing the performance of energy harvesting from vibration with low excitation level and wide frequency range.

However, it is challenging or even impossible in practice to obtain a nonlinear system with perfectly symmetric potentials. The reasons may come from the influence of the inhomogeneity of materials, the inconsistency of tip and external magnets, as well as the difficulties in modulating the configurations. Harne and Wang[29] indicated that a priori knowledge of the excitation environment and careful avoidance of asymmetries will benefit the energy harvesting using weakly bistable systems. Halvorsen[30] considered an asymmetric quartic potential in bistable system and indicated that it have a significant impact on the output. Moreover, He and Daqaq[31] explored an asymmetric potential similar to Halvorsen, their results under white noise excitations shown that the presence of asymmetries in MEHs serves to improve performance, while it has an adverse influence on BEHs especially for small to moderate white noise intensity. Although, there are rarely investigations into the influence mechanism of the asymmetric potentials, especially the corresponding strategy for improving the performance of asymmetric nonlinear systems.

Therefore, this paper concentrates on the problem of asymmetric potentials based on the piezoelectric bistable energy harvesters. Numerical simulations firstly demonstrate that asymmetries in potential function export a negative effect on the power output. Then the corresponding performance enhancement method by compensating the asymmetric potentials with a proper bias angle of the system is proposed and demonstrated numerically and experimentally. In Sect. 2, the 
configuration and the model of the $\mathrm{BEH}$ are presented. Then, the influence mechanism of the asymmetric potential energy function is numerically studied in Sect. 3. In addition, the performance enhancement method is proposed and proved. In Sect. 4, experimental verification of two bistable harvesters is carried out. Conclusions are followed in the final Sect. 5.

\section{Configuration and model of the bistable harvester}

Figure 1 illustrates the diagram of the nonlinear energy harvester investigated in this paper. The configuration is composed of a stainless steel substrate, two PZT piezoelectric layers at the root, tip magnests and external magnets. Due to the interaction of end magnets with external ones, the configuration could be modulated to bistable systems with different potential energy functions by adjusting the parameters $h, d$, and $\vartheta$.

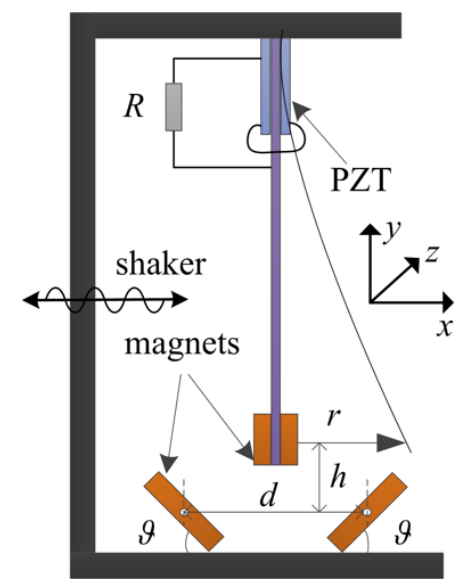

Fig.1 Schematic of the bistable energy harvester

When the external magnets are removed, the system will become a linear harvester. For the linear system, it is demonstrated that the variational indicator $\left(V^{\mathrm{N}}\right)$ should be zero all the time through Lagrange function[32, 33], just as

$V^{\mathrm{I}}=\int_{t_{1}}^{t_{2}}\left[\delta E_{\mathrm{k}}-\delta E_{\mathrm{p}}+E_{\mathrm{a}}\right] \mathrm{d} \bar{t}=0$

where $\delta$ is the variational symbol. And $E_{\mathrm{k}} 、 E_{\mathrm{p}} 、 E_{\mathrm{a}}$ respectively represent the kinetic energy, potential energy and the external work applied to the system. Based on Euler-Bernoulli beam theory, 
Rayleigh-Ritz method, and assumed-modes method, the electromechanical model[19] of the linear system under base excitation is $\left\{\begin{array}{l}m \ddot{r}(\bar{t})+c \dot{r}(\bar{t})+k r(\bar{t})-\theta \bar{V}(\bar{t})=\bar{F} \cos (\bar{\Omega} \bar{t}) \\ C_{\mathrm{p}} \dot{\bar{V}}(\bar{t})+\bar{V}(\bar{t}) / R+\theta \dot{r}(\bar{t})=0\end{array}\right.$

where $m, c, k$ are respectively the equivalent mass, damping and stiffness. $C_{\mathrm{p}}$ and $\theta$ are load resistance and equivalent electromechanical coupling coefficient. $\bar{V}$ is the output voltage and $r$ is the tip displacement. And $\bar{F}$ is the amplitude of the excitation force and $\bar{\Omega}$ is the frequency. Moreover, the dot represents a derivative with respect to time $\bar{t}$. For a nonlinear bistable system, nonlinear restoring force $\bar{F}_{\mathrm{h}}$ is applied to replace the linear force $k r$ in the model. Thus, the electromechanical model of the BEHs can be obtained as follows:

$\left\{\begin{array}{l}m \ddot{r}(\bar{t})+c \dot{r}(\bar{t})+\bar{F}_{\mathrm{h}}-\theta \bar{V}(\bar{t})=\bar{F} \cos (\bar{\Omega} \bar{t}) \\ C_{\mathrm{p}} \dot{\bar{V}}(\bar{t})+\bar{V}(\bar{t}) / R+\theta \dot{r}(\bar{t})=0\end{array}\right.$

For typical bistable systems, the nonlinear restoring force could be given as a polynomial form $\bar{F}_{\mathrm{h}}=-k_{1} \bar{x}+k_{3} \bar{x}^{3}$, where $k_{1}$ and $k_{3}$ are the coefficient of the restoring force. For brevity, the equations of motion can be further non-dimensionalized by introducing the following equations:

$x=\frac{r}{l_{c}}, t=\bar{t} \omega_{n}, V=\frac{C_{p}}{\theta l_{c}} \bar{V}$

where $l_{\mathrm{c}}$ is a length scale introduced to non-dimensionalized the displacement, $\omega_{n}=\sqrt{k_{1} / m}$ is the natural frequency of the harvester. With these transformations, the non-dimensional model can be expressed as

$\left\{\begin{array}{l}\ddot{x}+2 \xi \dot{x}-x+\delta x^{3}-\kappa^{2} V=F \cos (\omega t) \\ \dot{V}+\alpha V+\dot{x}=0\end{array}\right.$

where 
$\xi=\frac{c}{2 \sqrt{k_{1} m}}, \delta=\frac{k_{3} l_{c}^{2}}{k_{1}}, \kappa^{2}=\frac{\theta^{2}}{k_{1} C_{p}}$

$\alpha=\frac{1}{\omega_{n} C_{p} R}, F=\frac{\bar{F}}{k_{1} l_{c}}, \omega=\frac{\bar{\Omega}}{\omega_{n}}$.

Here, $x, V$ and $\omega$ are respectively the non-dimensional displacement, voltage and frequency. $\xi$ is the damping ratio. Finally, $F_{\mathrm{h}}=-x+\delta x^{3}$ is the non-dimensional restoring force and $\delta$ is the coefficient of cubic nonlinearity. In above equations, the bistable system has a perfectly symmetric potential energy function. To characterize the asymmetric potentials of the BEHs in this paper, a quadratic nonlinearity $[30,31]$ is introduced to the nonlinear restoring force. Thus, the restoring force can then be described as

$F_{\mathrm{h}}{ }^{\prime}=-x+\beta x^{2}+\delta x^{3}$

where $\beta$ is the coefficient of the quadratic nonlinearity. For the system, the average output in certain range of time can be given by

$$
P=E\left[\alpha \times V^{2}(t)\right]
$$

\section{Numerical investigation of asymmetric potential function}

\subsection{Influence of asymmetric potentials in BEHs}

In this part, we firstly consider the nonlinear dynamics of a perfectly symmetric potential $\mathrm{BEH}$ with $\beta=0$, which will be then applied to compare with asymmetric ones. Numerical simulations are carried out by Runge-Kutta method to obtain the system response and non-dimensional parameters $\xi=0.0933, \delta=0.5495, \kappa^{2}=0.00184$, and $\alpha=0.4065$ are utilized. Bifurcation diagram, voltage response, frequency spectrum, phase orbit and poincare map are applied the analyze the nonlinear dynamics. 

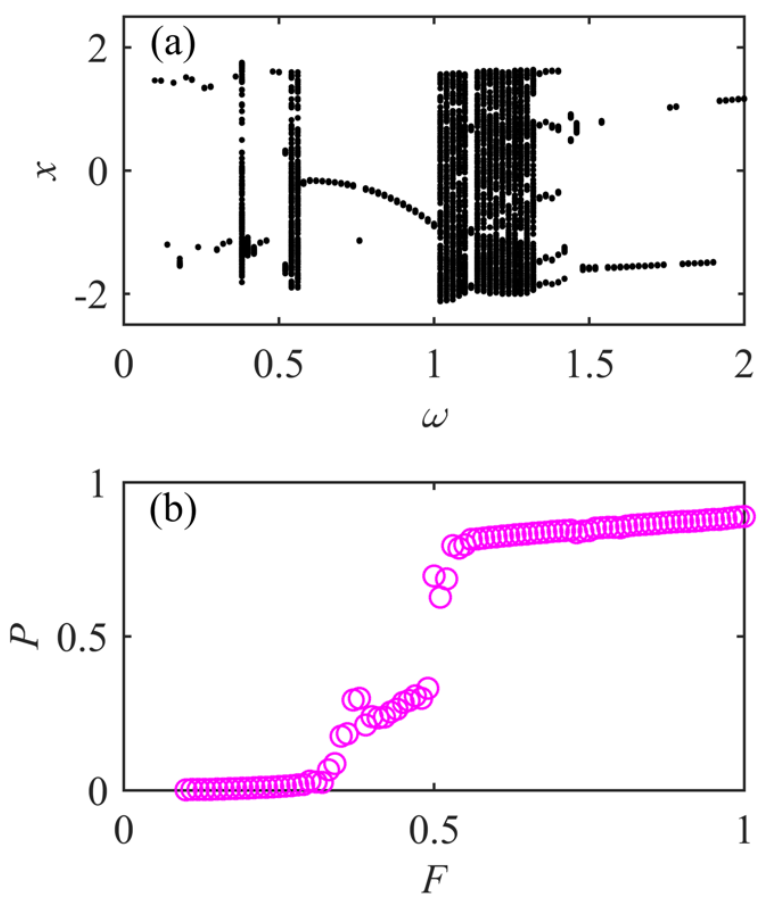

Fig.2 (a) Bifurcation of $x$ to $\omega$ for $F=0.5$; (b) bifurcation of output power to $F$ for $\omega=1$.

Figure $2 \mathrm{a}$ shows the bifurcation diagram of non-dimensional displacement $x$ varying along with the change of excitation frequency $\omega$. The excitation level $F$ in this case equals to 0.5 . It can be observed that the excitaion frequency exports a significant influence on the output response of the system. When $\omega$ is less than 0.56 , the response of the system is period- 1 motion in a single potential well except for several frequency 0.38 and 0.54 . Figure $3 \mathrm{a}$ illustrates the voltage response, frequency spectrum, phase orbit and poincare map when $\omega$ is 0.5 . It can be clearly viewed that the response is a small amplitude in a potential well, which also shows higher harmonic frequencies in $\omega=1$ and 1.5. As the frequency increases, the response of the system is inter-well oscillation in a high-energy branch[13]. Figure $3 \mathrm{~b}$ shows the corresponding large amplitude motion with a excitaion frequency 0.75 . With further increase of $\omega$ to the range from 1.02 to 1.32 , the response of the system is chaotic oscillation. It is observed from Figure 4a that there is strange attractors in the poincare map. Moreover, the response frequencies of the output voltage are distributed in a wide range. When $\omega$ increases from 1.32, the system will enter into periodic motion from chaos. Figure 
$4 \mathrm{~b}$ shows the voltage response, frequency spectrum, phase orbit and poincare map when $\omega$ equals to 1.4. It is seen that the response of the BEH becomes period-5 oscillation across the potential wells and there are five isolated points in the poincare map. For excitation frequency larger than 1.42 , the response of BEH will again enter into a single potential well, generating smaller output power.

(a)
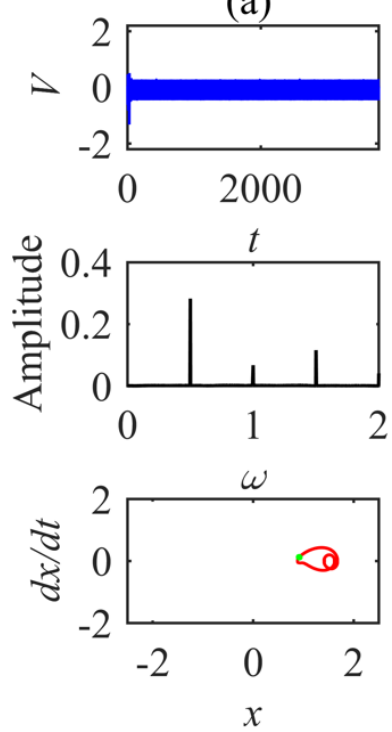

(b)
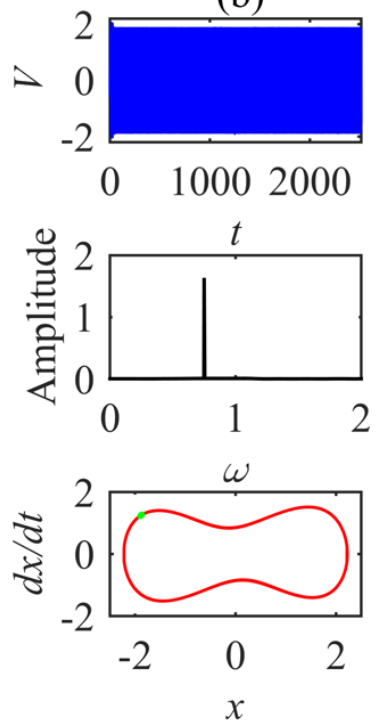

Fig.3 Voltage response, frequency spectrum, phase orbit and poincare map of the BEH for $\omega=0.5$ and $\omega=0.75$ when $F$ equals to 0.5

(a)
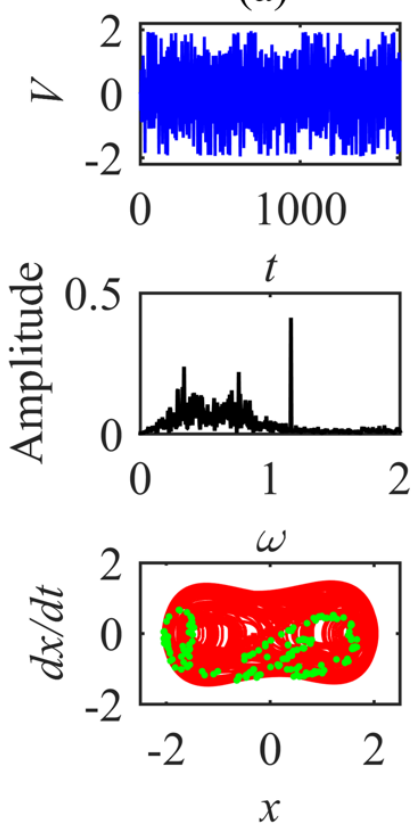

(b)
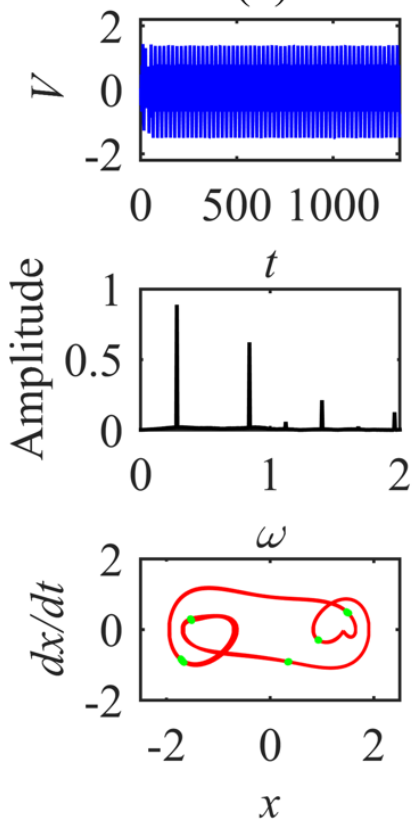

Fig.4 Voltage response, frequency spectrum, phase orbit and poincare map of the BEH for $\omega=1.16$ and $\omega=1.4$ when $F$ equals to 0.5 
For characterizing the influence of excitation level on the output power of the BEH, the bifurcation diagram of average power calculated from Eq. (8) to $F$ is obtained in Figure $2 b$ for $\omega=1.0$. In this case, the excitation level is ranged from 0.1 to 1.0 . When $F$ is smaller than 0.32 , the output power of the system is very small for the reason that the response is in intra-well motion state. With the increase in $F$, there is enough energy for the system to overcome the potential barrier, but it firstly shows chaotic oscillation for excitation level below 0.53 . For $F$ beyond 0.53 , the BEH obtains large-amplitude inter-well motion to output more electric energy, and the energy shows an increasing trend with the increase of excitation amplitude.

In order to investigate the influence of asymmetric potential function on the response of BEHs, the quadratic nonlinearity in the restoring force is considered for different values with $\beta$ equaling to $0.15,0.25$, and 0.35 . The corresponding potential energy functions of the three asymmetric potential BEHs are shown in Figure 5. It is seen that the potential well on the left becomes much deeper, while the right one shallower with the increase of the value of $\beta$.

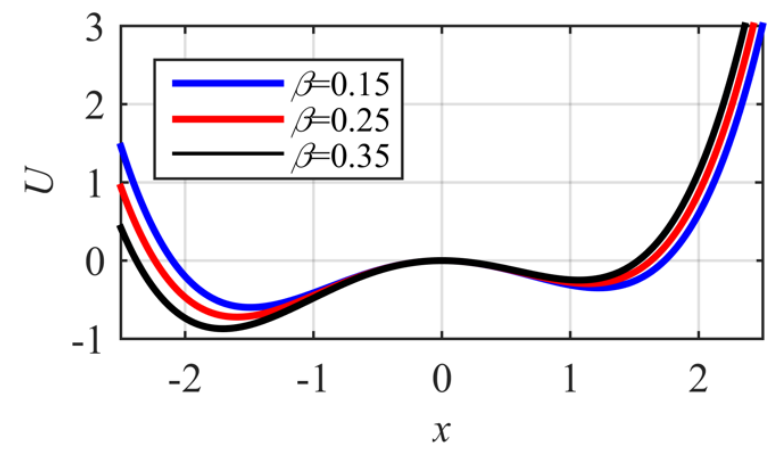

Fig.5 Potential energy functions of three asymmetric BEHs when $\beta$ equals to $0.15,0.25$, and 0.35 .

Under excitation level 0.5 , the bifurcation diagram of $x$ to $\omega$ for the three asymmetric potential BEHs are respectively illustrated in Figure $6 \mathrm{a}, \mathrm{b}$ and $\mathrm{c}$. For $\beta$ equaling to 0.15 , the BEH oscillates in the high-energy branch in the frequency range from 0.62 to 1.0. It should be noted that for some special frequencies in this range, the nonlinear oscillation is in intra-well state. When the frequency 
varies from 1.0 to 1.38 , the oscillation of the $\mathrm{BEH}$ shows chaotic characteristics. For other frequencies less than 0.62 and larger than 1.38, the motions of the BEH are also kept in a single well. As $\beta$ increases to 0.25 , the frequency range for chaotic oscillation becomes narrower from 1.08 to 1.38, while the two cut-off frequencies respectively 0.66 and 1.38 . In the range from 0.66 to 1.08 , there are more frequencies resulting in a small amplitude motion in intra-well, while less for the inter-well motion. When $\beta$ is increased to 0.35 , the BEH will oscillate chaotically in a much narrower frequency range from 1.18 to 1.36 . Besides several specific frequencies resulting in inter-well motion, the BEH vibrates in low-energy branch for other frequencies less than 1.18 and larger than 1.36. Furthermore, when there are asymmetries in BEHs, it has a higher probability for the system to oscillate in the deeper potential wells for intra-well motion.
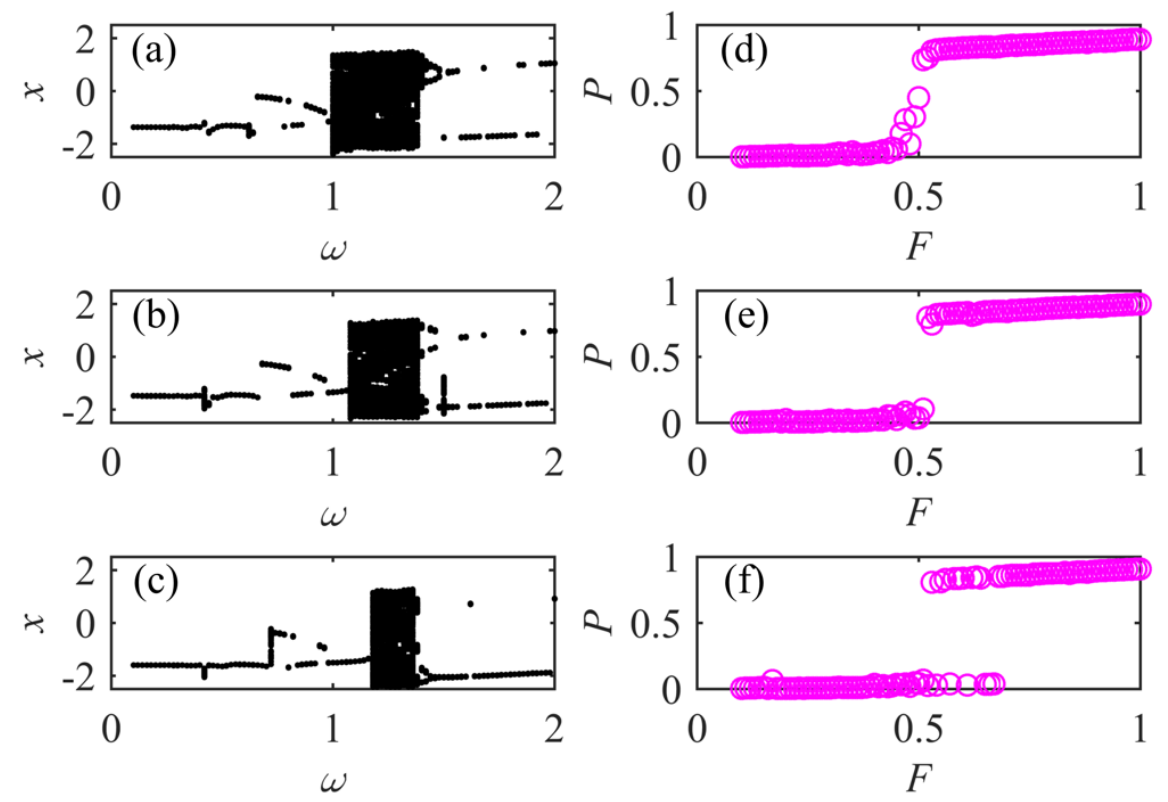

Fig.6 Bifurcation of $x$ to $\omega$ for $F=0.5$ and bifurcation of output power to $F$ for $\omega=1$ for different asymmetric potential BEH: (a) and (d) for $\beta=0.15$; (b) and (e) for $\beta=0.25$; (c) and (f) for $\beta=0.35$.

Figure $6 \mathrm{~d}$ shows the bifurcation diagram of average power varying with $F$ for $\beta$ equaling 0.15 . When compared with the results in Figure $2 b$, it is observed that the smallest excitation level for the chaotic increases to 0.45 , and only in a narrower range from 0.45 to 0.53 . While the excitation level for large amplitude inter-well motion is not influenced. With $\beta$ increasing to 0.25 , the oscillation of 
the BEH for $F$ less than 0.51 is always in a single well, and the motion is in high-energy branch when $F$ larger than 0.52 . As $\beta$ increases to 0.35 , although the smallest excitation level for inter-well motion is not influenced, but there are still values for intra-well oscillation until $F$ increases to 0.67 . Comparing these results to that of symmetric $\mathrm{BEH}$, it is demonstrated that the presence of asymmetries in BEHs will export negative impact on the response of nonlinear bistable energy harvesting systems, and this impact will be aggravated with the increase of asymmetric degree.

\subsection{Performance enhancement method}

From the simulations above, it is concluded that the presence of asymmetries in BEHs have negative impact on the response of the system. In this part, it is supposed that the efficiency of the asymmetric potential BEHs could be enhanced by compensating the asymmetric potentials to symmetric ones and this result can be obtained by applying external forces to the energy harvesters or wisely using the self-gravity. In this paper, a proper bias of the asymmetric potential BEHs is introduced. In this case, the asymmetric potential functions could be compensated to be symmetric by the gravity of the cantilever beam and this motivation comes from the application of BEHs to harvest energy from the swing motion of human lower-limb[14]. For the system with a bias angle, the corresponding electromechanical model could be expressed as following[14]

$$
\left\{\begin{array}{l}
\ddot{x}+2 \xi \dot{x}-x+\beta x^{2}+\delta x^{3}-p \sin \phi-\kappa^{2} V=F \cos (\omega t) \\
\dot{V}+\alpha V+\dot{x}=0
\end{array}\right.
$$

where $p$ is the equivalent non-dimensional gravity of the cantilever beam, $\phi$ is the bias angle of the system and $F_{\mathrm{h}}{ }^{\prime}=-x+\beta x^{2}+\delta x^{3}-p \sin \phi$ is the corresponding nonlinear restoring force. In this paper, $\phi$ is assumed to positive for clockwise angle and negative otherwise.

Based on the equations, it is supposed that there is an optimum bias angle for the system to 
output largest power, under which a symmetric potential BEH is obtained. To obtained the value, the derivative of the nonlinear restoring force is assumed to be zero and its two solutions are respectively $x_{1,2}=-\beta / 3 \delta \pm \sqrt{\beta^{2}+3 \delta} / 3 \delta$

To make the asymmetric BEHs be a symmetric one, it will satisfy the following equation

$F_{\mathrm{h}}^{\prime}\left(x_{1}\right)+F_{\mathrm{h}}^{\prime}\left(x_{2}\right)=0$

In this way, the optimum bias angle of the system is obtained as

$$
\phi=\arcsin \left(\frac{2 \beta^{3}+9 \beta \delta}{27 p \delta^{2}}\right)
$$
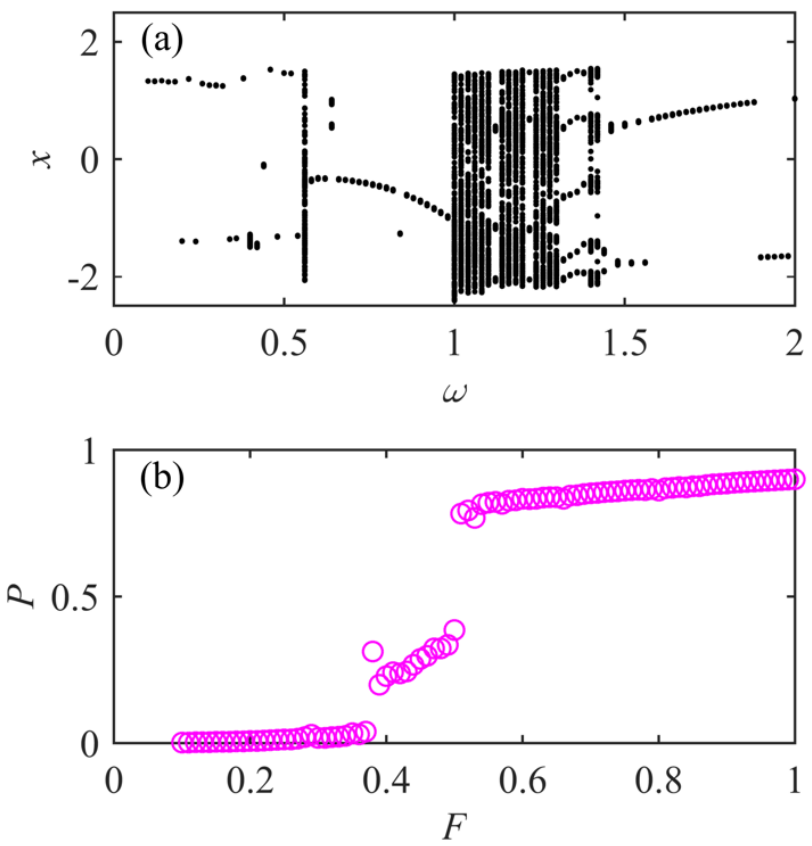

Fig.7 (a) Bifurcation of $x$ to $\omega$ for $F=0.5$ and (b) bifurcation of output power to $F$ for $\omega=1$ when the asymmetric $\mathrm{BEH}(\beta=0.25)$ is balanced with a certain angle.

To illustrate the effectiveness of the proposed performance enhancement method, the asymmetric potential $\mathrm{BEH}$ with $\beta=0.25$ is considered. Bifurcation diagrams of displacement varying with $\omega$ as well as average output power varying with $F$ shown in figure 7 are applied to compare with the results of asymmetric one. Compared with the results before, it is observed from figure $7 \mathrm{a}$ that the frequency range for high-energy branch oscillation becomes wider from 0.56 to 1.0 . When $\omega$ is less than 0.56 , the oscillation of the cantilever is kept in a single well, thus generating less 
electricity. For frequencies larger than 1.42, the response of the BEH is also in intra-well state. As the frequency increases from 1.0 to 1.30 , there exist strange attractors and the response is chaotic in inter-well state. What's more, it is seen that the period-5 inter-well motion is achieved in the frequency range from 1.30 to 1.42 , which is much wider than the results of asymmetric BEH. From the bifurcation diagrams of average output power varying with $F$ shown in figure $7 \mathrm{~b}$, it is seen that the $\mathrm{BEH}$ begin to travel across the potential wells when the $F$ increases to 0.37 and obtained the large-amplitude high-energy branch from $F$ equaling to 0.52 . By comparing the results with that of symmetric $\mathrm{BEH}$, it is demonstrated that the performance of the asymmetric bistable energy harvester is enhanced by introducing a proper bias angle to the system.
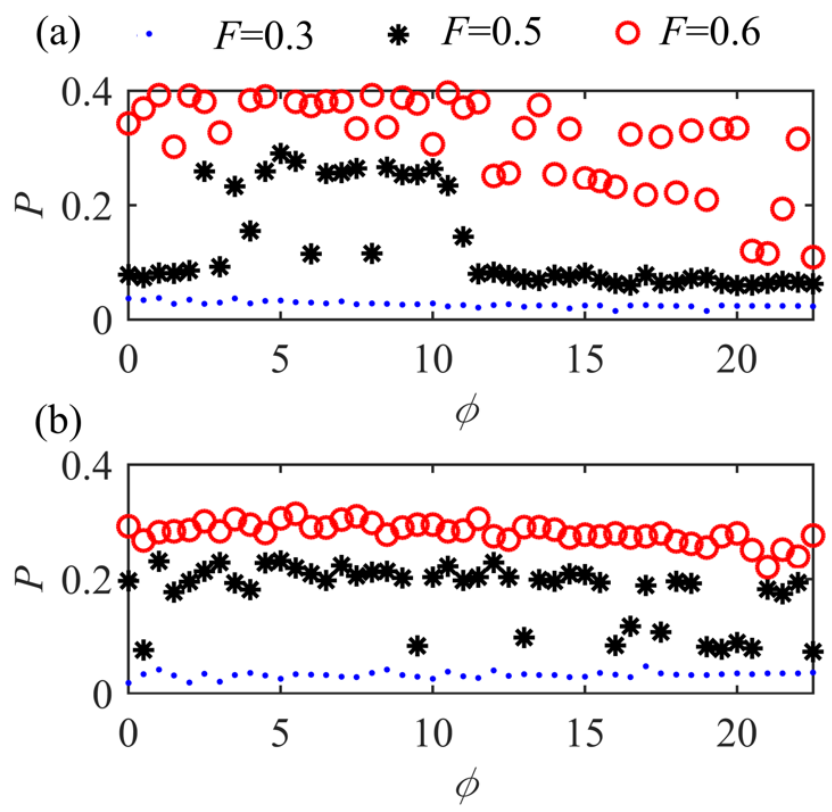

Fig.8 Output power of the BEH with $\beta=0.15$ under different bias angles for up-(a) and down-sweep (b) frequency excitations.

What's more, the three asymmetric potential BEHs with $\beta$ equaling to $0.15,0.25$ and 0.35 are simulated under up- and down-sweep frequency excitations under different acceleration levels and bias angles. The range of $\omega$ considered here is from 0.1 to 2.0 and the bias angle $\phi$ ranges from 0 to $\pi / 8$. The average output powers under different excitations are calculated and the results for the 
three asymmetric potential BEHs are respectively shown in figure 8, 9 and 10.
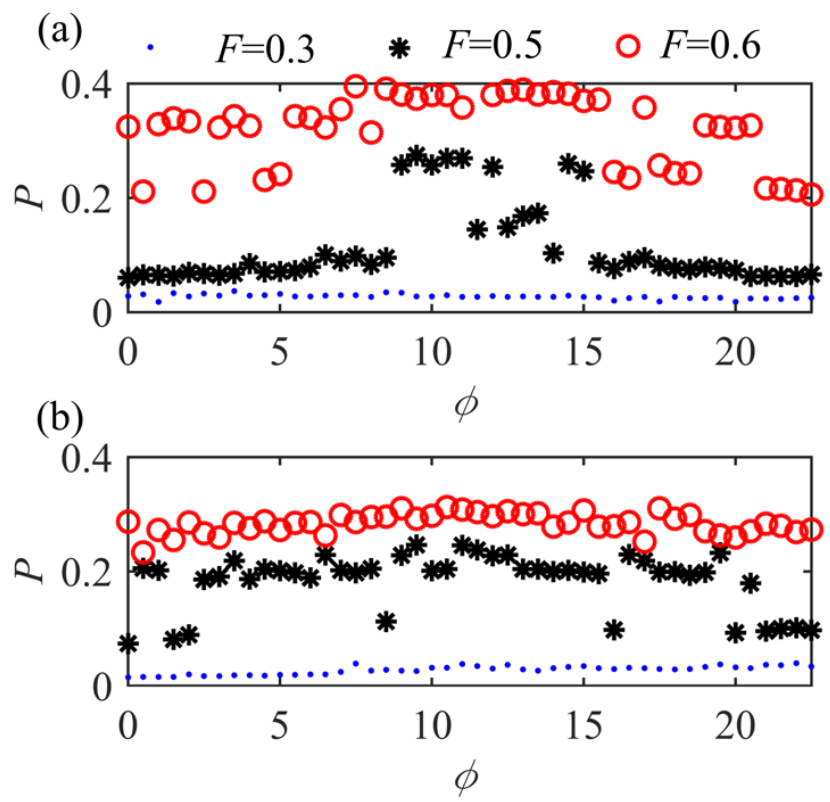

Fig.9 Output power of the BEH with $\beta=0.25$ under different bias angles for up- (a) and down-sweep (b) frequency excitations.
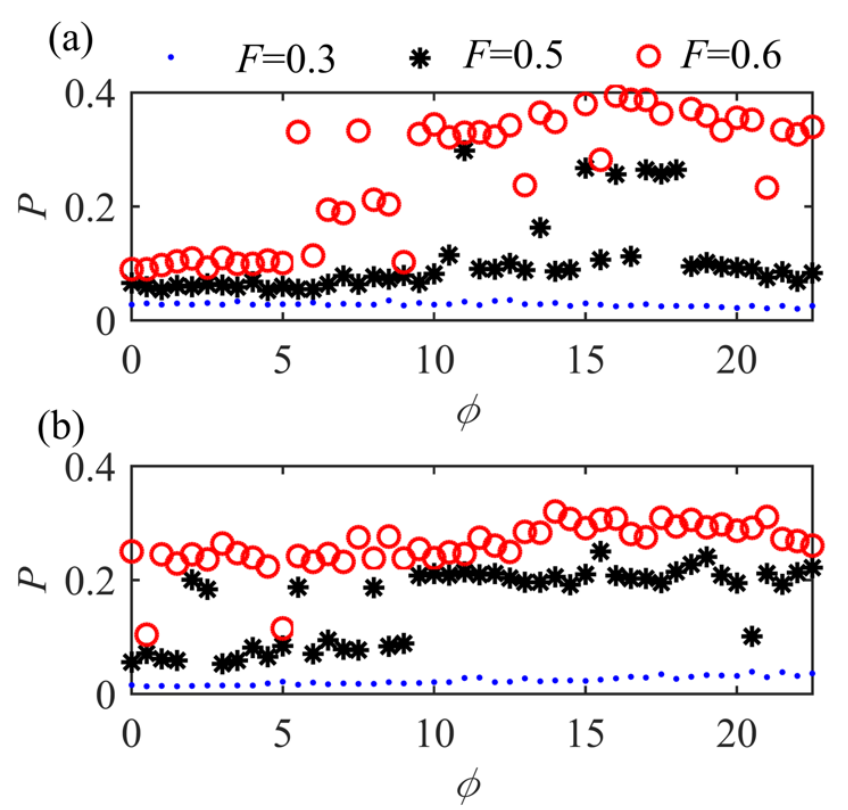

Fig.10 Output power of the BEH with $\beta=0.35$ under different bias angles for up- (a) and down-sweep (b) frequency excitations.

Under lower excitation level $F=0.3$, it is observed that the average output power of the three BEHs are almost unaffected by the bias angle for the reason that the oscillation of the BEH is always in intra-well state. Under excitation level 0.5 , there is an optimum bias angle range from $2.5^{\circ}$ to 
$10.5^{\circ}$ for the asymmetric potential $\mathrm{BEH}$ with $\beta=0.15$ to travel across the potential wells to generate larger output power under up-sweep frequency excitation. With $\beta$ increasing to 0.25 , it is noticed that the optimum bias angle range for up-sweep frequency excitation becomes narrower and moves to larger values from $9^{\circ}$ to $15^{\circ}$. For down-sweep frequency excitation, the two asymmetric potential BEHs can almost generate larger output power except for several isolated angles. As $\beta$ is increased 0.35 , the range for up-sweep frequency excitation becomes much narrower and the BEH only generates larger power around $17.5^{\circ}$. Interestingly, it is viewed that the BEH under down-sweep frequency excitation begins to generate larger output power when the bias angle increases to $9.5^{\circ}$ for larger asymmetric degree with $\beta=0.35$. For largest excitation level 0.6 , the asymmetric potential BEH with $\beta=0.35$ begins to travel across the potential well and generates larger power from bias angle $9.5^{\circ}$, while for other asymmetric degrees the output power are almost unaffected whether for up or down sweep frequency excitation. Based on the results, it can be demonstrated that there exists an optimum bias angle range for asymmetric potential BEHs to generate larger output power and the range is influenced by excitation levels and asymmetric degrees.

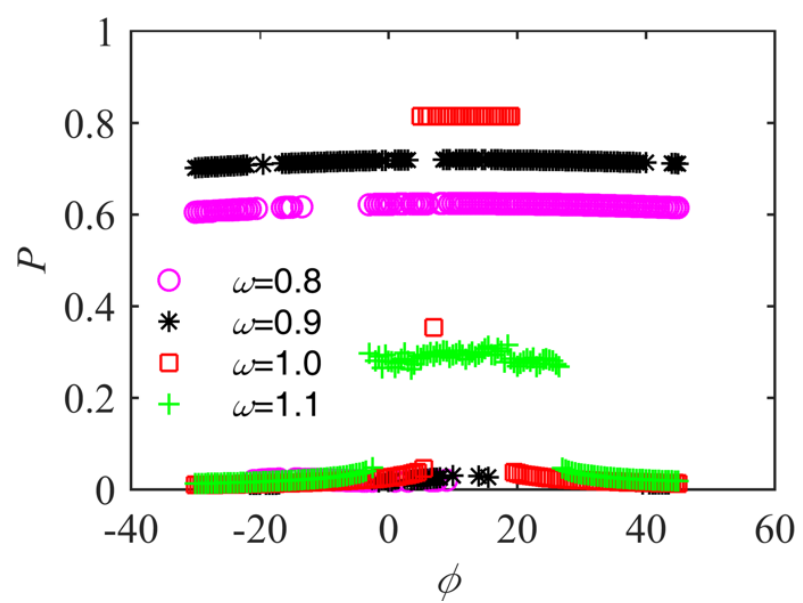

Fig.11 Output power of BEH with $\beta=0.25$ under different bias angles for different $\omega$ when $F=0.5$. For asymmetric potential BEH with $\beta=0.25$, the average output power under different excitation frequencies and bias angles are obtained and the results are illustrated in figure 11 . The asymmetric 
potential BEH oscillates on high-energy branch and generates larger output power in an optimum bias angle range around the optimum value. And the optimum range becomes narrower with the increase of $\omega$. Therefore, it is concluded that the performance of asymmetric potential BEHs could be enhanced by compensating the asymmetric potentials with a proper bias angle of the system. And the optimum bias angle range is influenced by the asymmetric degree caused by the quadratic nonlinearity in nonlinear restoring force.

\section{Experimental verification}

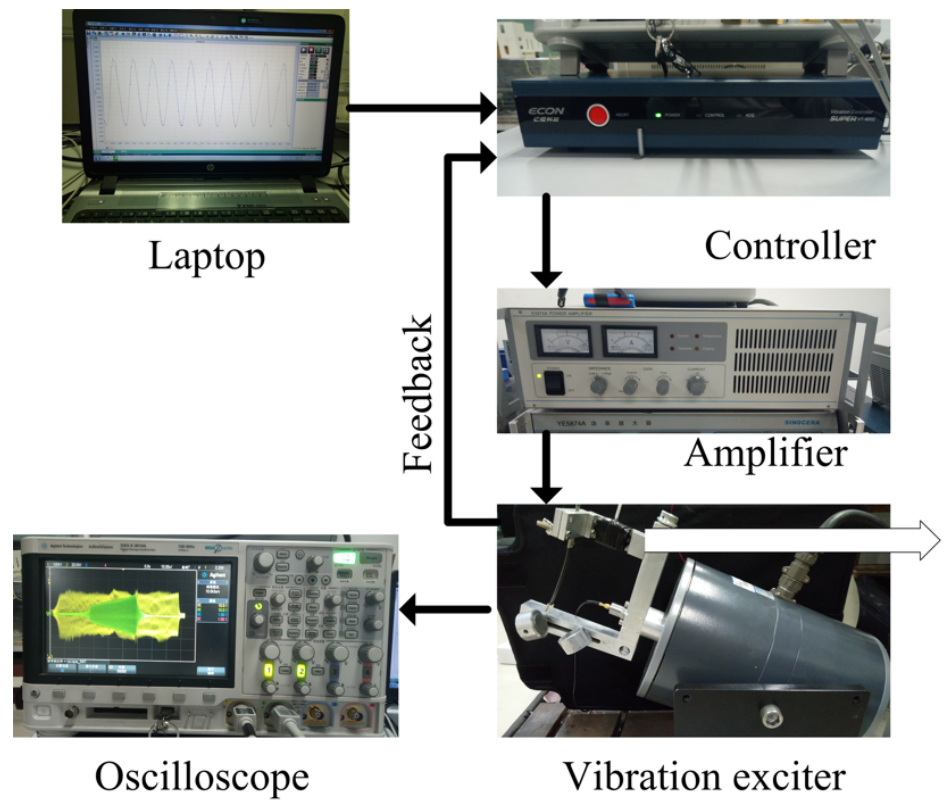

(a)

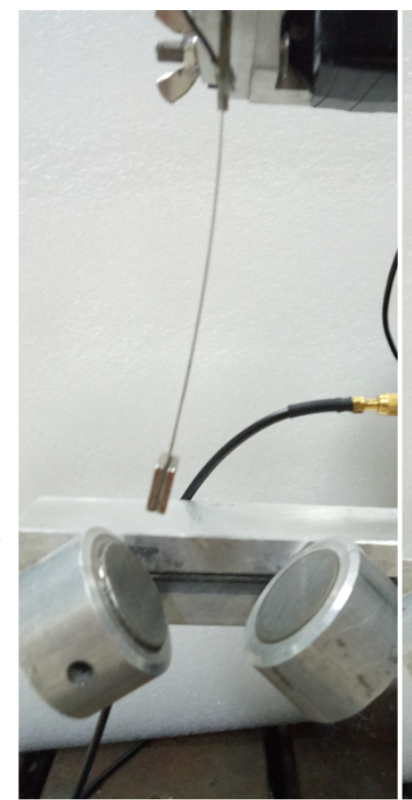

(b)

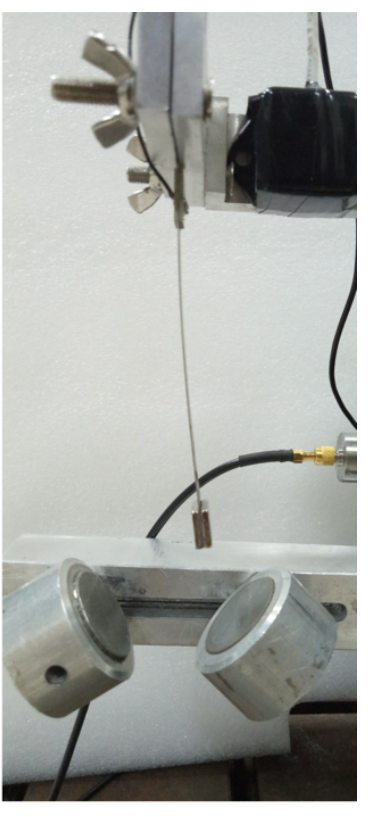

(c)

Fig.12 (a) Experimental setup; (b) and (c) are two stable equilibrium positions of the BEH.

To verify the effectiveness of the proposed method above, the experimental system shown in Figure 12(a) is set up. Bistable energy harvesters are installed on the vibration exciter (JZK-50). The bias angle of the system could be adjusted with the inclination of the exciter. The exciter is actuated by signal sources produced from a power amplifier (YE5874A) and a signal generator (VT-9002-1), which also acts as a controller. An acceleration sensor EA-YD-185 is applied to measure the acceleration and the experimental data are acquired by an oscilloscope (MSOX3052A) with probe 
resistance of $10 \mathrm{M} \Omega$. The middle substrate used in the experiments is made of stainless steel with the dimension of $92 \times 15 \times 0.27 \mathrm{~mm}^{3}$. Two piezoelectric layers made from PZT has the dimension of 12 $\times 10 \times 0.8 \mathrm{~mm}^{3}$. All magnets used in the experiments are $\mathrm{NdFeB}$ magnets and have the strength of N35. Two tip magnets have the dimension of $10 \times 10 \times 2 \mathrm{~mm}^{3}$ and the external magnets have the diameter of $25 \mathrm{~mm}$ and thickness of $5 \mathrm{~mm}$. Two bistable energy harvesters (BEH-1 and BEH-2) with different asymmetric degrees are obtained in the experiments and the two stable equilibria of the $\mathrm{BEH}$ at horizontal position are illustrated in Figure 12 (b) and (c). Also, Figure 13 shows the potential energy functions of the two BEHs. In the experiments, BEHs are tested at several specific bias angles of $-15^{\circ}, 0^{\circ}, 10^{\circ}, 20^{\circ}, 30^{\circ}, 45^{\circ}$ and $60^{\circ}$. For bias angles of $0^{\circ}, 10^{\circ}$ and $20^{\circ}$, BEH- 1 is still a bistable configuration, while it becomes a monostable one for other bias values. While for BEH-2, it is a monostable configuration for bias angles of $-15^{\circ}$ and $60^{\circ}$, and a bistable one for other values.

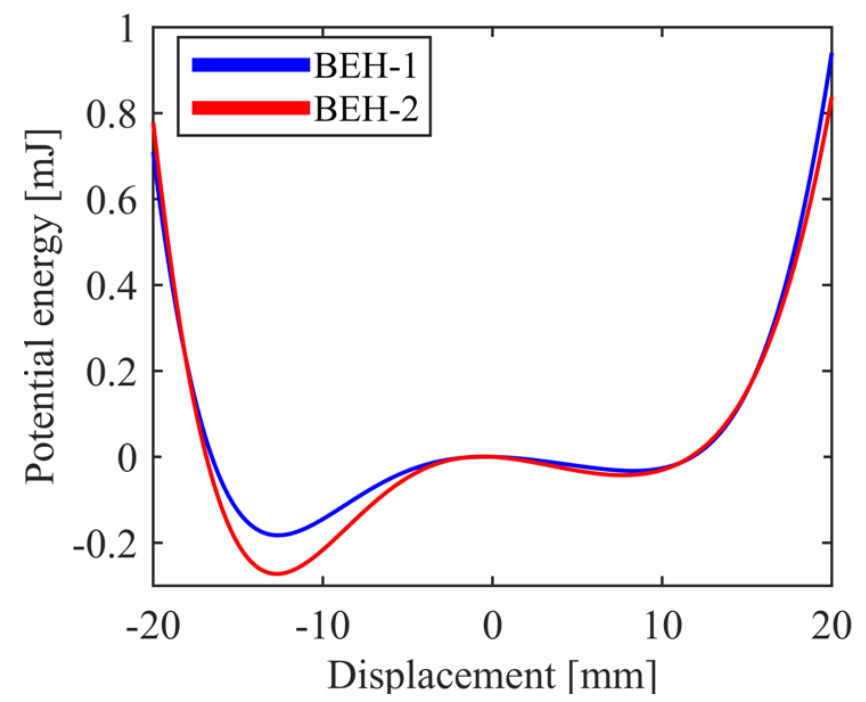

Fig.13 Potential energy function of BEH-1 and BEH-2 in the experiments 


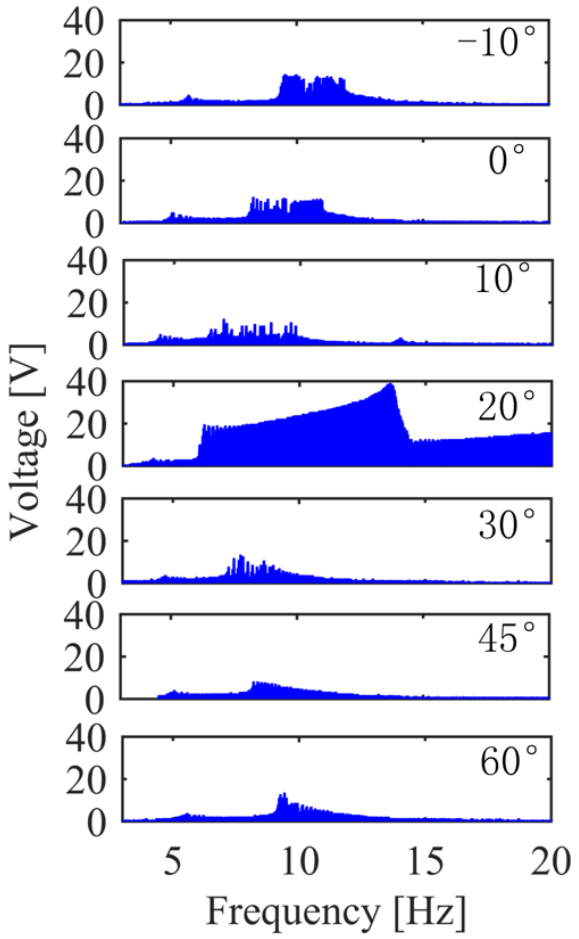

(a) Up-sweep
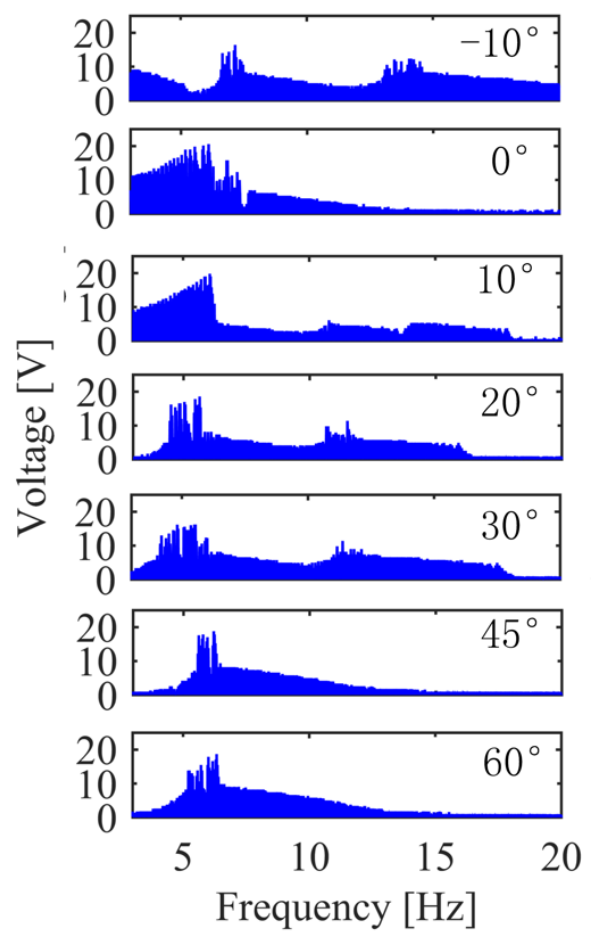

(b) Down-sweep

Fig.14 Voltage response of BEH-1 under up- and down-sweep frequency excitation for acceleration level of $3 \mathrm{~m} / \mathrm{s}^{2}$

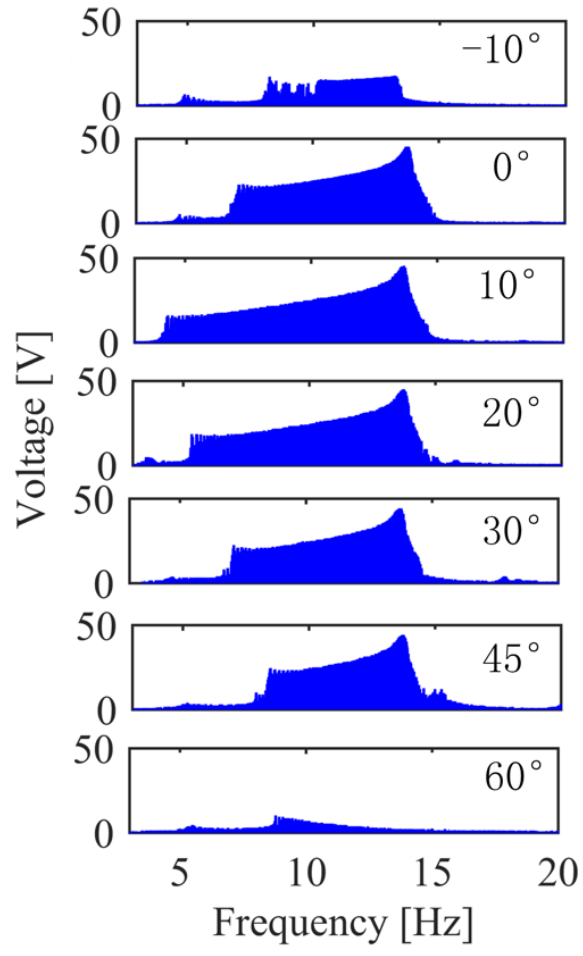

(a) Up-sweep

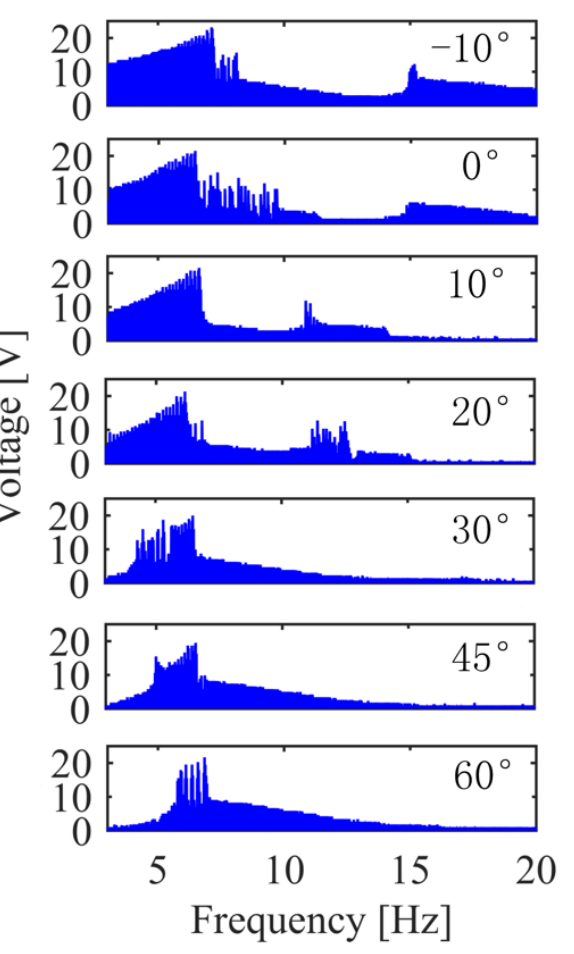

(b) Down-sweep

Fig.15 Voltage response of BEH-1 under up- and down-sweep frequency excitation for acceleration level of $4 \mathrm{~m} / \mathrm{s}^{2}$ 
Figure 14a shows the voltage response of BEH-1 under up-sweep frequency excitation of $0.3 \mathrm{~g}$ at different bias angles. For bias angles of $-15^{\circ}, 0^{\circ}, 10^{\circ}, 30^{\circ}, 45^{\circ}$ and $60^{\circ}$, the oscillation of BEH-1 is limited in a single potential well and it generates smaller electric energy. While for bias angle of $20^{\circ}$, BEH-1 achieves high-energy inter-well motion in a broadband frequency range from $6.2 \mathrm{~Hz}$ to 13.6 $\mathrm{Hz}$ and the maximum voltage reaches $39 \mathrm{~V}$. When the excitation level is increased to $0.4 \mathrm{~g}$, BEH-1 could do inter-well motion at more bias angles and the corresponding voltage responses under up-sweep frequency excitation are illustrated in figure 15a. It is observed that the frequency range for BEH-1 on high-energy branch firstly becomes wider with the increasing of bias angle, and then narrower. Finally, the state of BEH-1 is in a single well for bias angle of $60^{\circ}$. Among all bias angles, the widest frequency range is from $4.2 \mathrm{~Hz}$ to $14 \mathrm{~Hz}$ at an optimum bias angle of $10^{\circ}$ and the obtained maximum voltage is $45.5 \mathrm{~V}$. For down-sweep frequency excitation of $0.3 \mathrm{~g}$, the responses shown in figure $14 \mathrm{a}$ indicates that $\mathrm{BEH}-1$ only achieves periodic high-energy inter-well motion at bias of $0^{\circ}$ and $10^{\circ}$, with little difference in the frequency range. With bias angle increases from $20^{\circ}$ to $60^{\circ}$, the obtained inter-well motion is chaotic and the main frequency range shifts to higher frequencies due to the change in potential energy functions. With excitation level increased to $0.4 \mathrm{~g}$, the responses show similar variational trend, but bias angle for periodic inter-well motion is widen from $-10^{\circ}$ to $20^{\circ}$. 


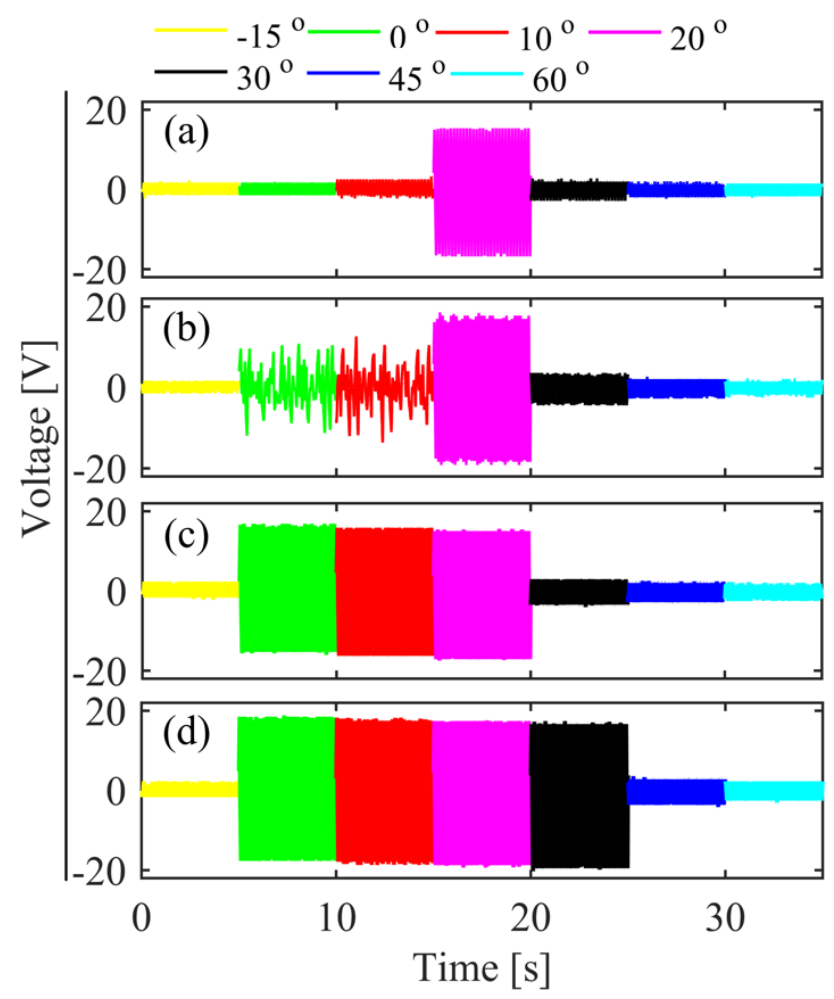

Fig.16 Voltage output of BEH-1 for different bias angles under constant frequency: (a) $0.3 \mathrm{~g}$, $6 \mathrm{~Hz}$; (b) $0.3 \mathrm{~g}, 7 \mathrm{~Hz}$; (c) $0.4 \mathrm{~g}, 6 \mathrm{~Hz}$; (d) $0.4 \mathrm{~g}, 7 \mathrm{~Hz}$.

For harmonic excitation with acceleration of $0.3 \mathrm{~g}$ and frequency $6 \mathrm{~Hz}$, the output voltages shown in figure 16a firstly increase and then decrease with an increase of the bias angle. At bias angle of $20^{\circ}$, BEH-1 exhibits periodic inter-well motion and a maximum voltage $15.4 \mathrm{~V}$ is obtained. With frequency increasing to $7 \mathrm{~Hz}$ (figure 16b), BEH-1 achieves large-amplitude inter-well oscillation and generates the largest voltage $18.45 \mathrm{~V}$ at bias of $20^{\circ}$, along with chaotic dynamics for bias of $0^{\circ}$ and $10^{\circ}$. For excitation of $0.4 \mathrm{~g}$ and $6 \mathrm{~Hz}$ (figure 16c), the oscillation of $\mathrm{BEH}-1$ is on high-energy branch for bias of $0^{\circ}, 10^{\circ}$ and $20^{\circ}$, while it is on the low-energy branch for other bias angles. Besides $0^{\circ}, 10^{\circ}$ and $20^{\circ}, \mathrm{BEH}-1$ also surfs high-energy branch for bias angle $30^{\circ}$ for a larger frequency of $7 \mathrm{~Hz}$ (figure 16d). The above experimental results are in agreement with numerical simulations, demonstrating that balancing the asymmetric potential with proper bias angles could enhance the performance of an asymmetric bistable harvester. 


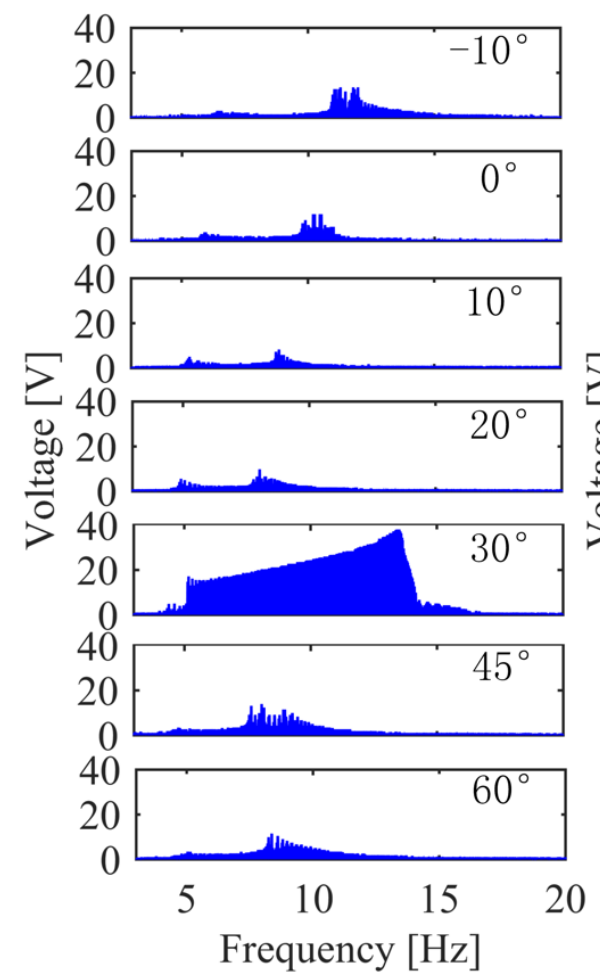

(a) Up-sweep

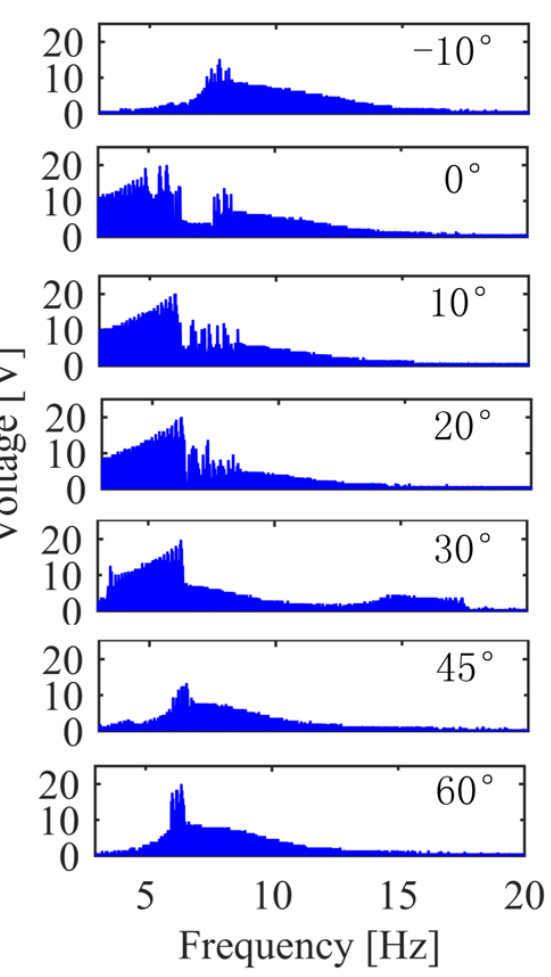

(b) Down-sweep

Fig.17 Voltage response of BEH-2 under up- and down-sweep frequency excitation for acceleration level of $3 \mathrm{~m} / \mathrm{s}^{2}$

For BEH-2 with larger asymmetric degree, the voltage responses under up-sweep frequency excitation of $0.3 \mathrm{~g}$ at different bias angles are shown in figure $17 \mathrm{a}$. It can be observed that the harvester explores a high-energy inter-well oscillation from $5.2 \mathrm{~Hz}$ to $13.5 \mathrm{~Hz}$ at bias angle of $30^{\circ}$, with a maximum output voltage of $38 \mathrm{~V}$. For other bias angles, the energy harvester is in intra-well state. As acceleration level increases to $0.4 \mathrm{~g}$ (figure 18a), the bias angles for large-amplitude inter-well motion are wider from $10^{\circ}$ to $45^{\circ}$, with a widest frequency range from $5.36 \mathrm{~Hz}$ to $13.65 \mathrm{~Hz}$ and maximum output voltage at $30^{\circ}$. For down-sweep frequency excitations shown in figure $17 \mathrm{~b}$ and figure $18 \mathrm{~b}$, the frequency range for inter-well oscillation also moves to larger frequencies similar to BEH-1 due the potential energy functions. 


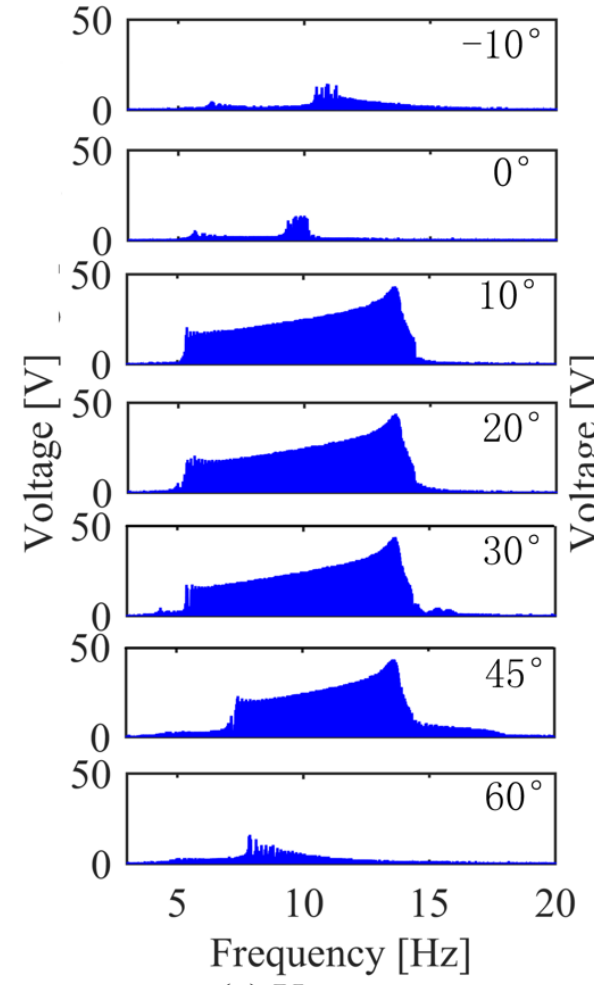

(a) Up-sweep
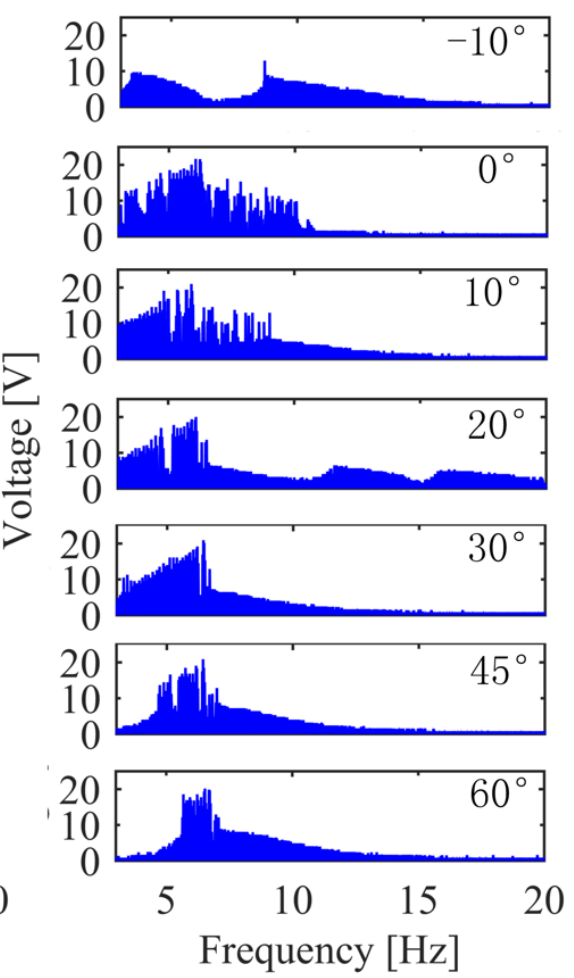

(b) Down-sweep

Fig.18 Voltage response of BEH-2 under up- and down-sweep frequency excitation for acceleration level of $4 \mathrm{~m} / \mathrm{s}^{2}$

Under constant frequency excitation of $6 \mathrm{~Hz}$ and $0.3 \mathrm{~g}$, the motion of BEH-2 shown in figure 19a is always in a single potential well for the reason there is insufficient energy for the system to achieve the high-energy branch. When the frequency increases to $7 \mathrm{~Hz}$, the harvester can travel across the potential wells for bias angle of $30^{\circ}$, with a maximum voltage of $22.17 \mathrm{~V}$ generated. Similarly, the optimum bias angle is also $30^{\circ}$ for excitation of $0.4 \mathrm{~g}$ and $6 \mathrm{~Hz}$. Besides bias angle of $30^{\circ}$, the oscillation of BEH-2 is also on high-energy branch at $20^{\circ}$ and $45^{\circ}$ for harmonic excitation of $0.4 \mathrm{~g}$ and $7 \mathrm{~Hz}$. Comparing the above results with that of BEH-1, the optimum bias angle range for BEH-2 with larger asymmetric degree moves to larger values, which is in agreement with the numerical simulations. Furthermore, it is demonstrated the bias angle range has close relationship with the excitation frequency and acceleration. 


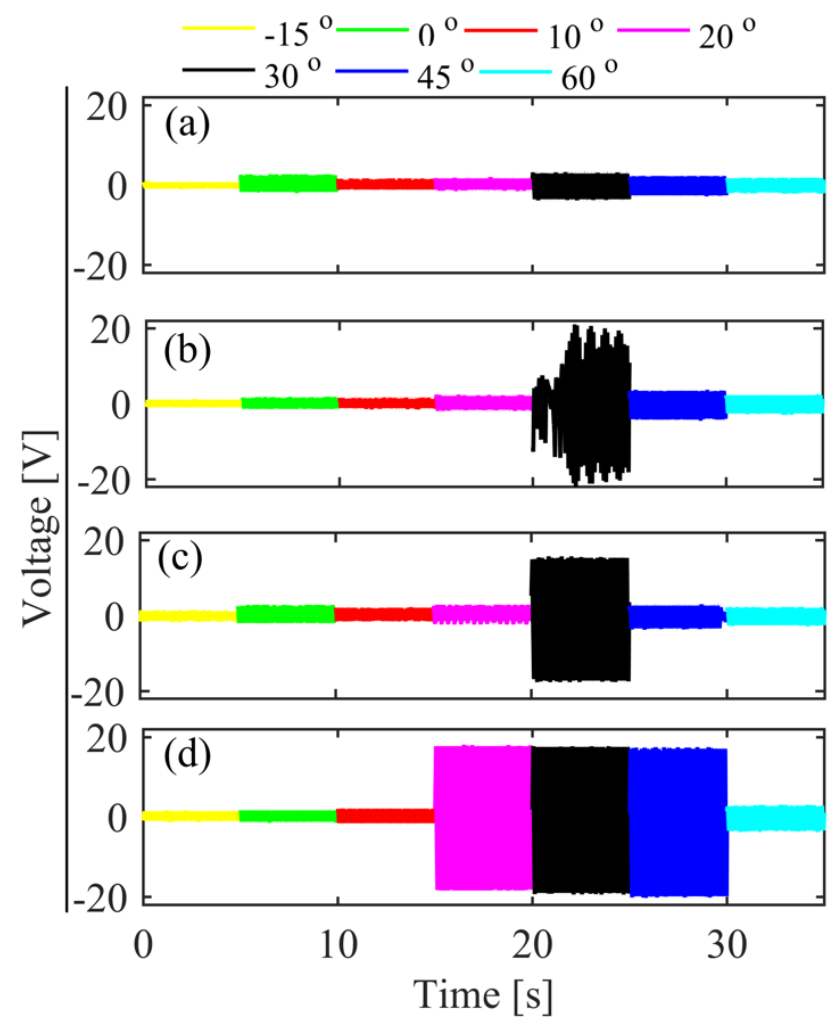

Fig.19 Voltage output of BEH-1 for different bias angles under constant frequency: (a) 0.3g, 6Hz; (b)

$$
0.3 \mathrm{~g}, 7 \mathrm{~Hz} \text {; (c) } 0.4 \mathrm{~g}, 6 \mathrm{~Hz} \text {; (d) } 0.4 \mathrm{~g}, 7 \mathrm{~Hz} \text {. }
$$

\section{Conclusion}

This paper investigates the influence of asymmetric potential energy functions on the nonlinear dynamics of bistable energy harvesters and proposes a performance enhancement method. A quadratic nonlinearity is introduced to the nonlinear restoring force to characterize the asymmetric degree of the bistable energy harvesters. Bifurcation diagrams from numerical simulation of the dimensionless electromechanical equation indicate that the presence of asymmetries in bistable energy harvesters have a negative impact on the output response. With the increase of the asymmetric degree, the frequency range for periodic and chaotic inter-well oscillation becomes narrower, which greatly limit the output power of bistable energy harvesters.

A performance enhancement method is proposed by compensating the asymmetric potentials with a proper bias angle of the asymmetric bistable system, which is motivated by harvesting energy 
from the swing motion of human lower-limb. The optimum bias angle for performance enhancement is derived and bifurcation diagrams of the enhanced bistable energy harvester demonstrate the effectiveness of the proposed method. Also, numerical simulations under sweep and constant frequency excitations demonstrate that the optimum bias angle range will move to larger values with the increase of asymmetric degrees, which is in agreement with the analytical results. Experimental results of two bistable energy harvesters with different asymmetric degree verify that the performance of asymmetric bistable harvesters would be enhanced by inclining the system with a certain bias angle. It is also observed in the experiments that the optimum bias angle range has close relationship with the asymmetric degree, as well as the excitation acceleration and frequency.

Acknowledgment This study has been supported by the National Natural Science Foundation of China (Grant No. 51575426, 51421004, 51611530547); Novel Energy Materials, Engineering Science and Integrated Systems (NEMESIS)(ERC Grant No. 320963) and Fundamental Research Funds for the Central Universities of China (Grant No. xjj2016002). 


\section{References}

1. Harne, R.L., Wang, K.W.: A review of the recent research on vibration energy harvesting via bistable systems. Smart Materials and Structures. 22(2), 023001(2013)

2. Pellegrini, S.P., Tolou, N., Schenk, M., Herder, J.L.: Bistable vibration energy harvesters: A review. Journal of Intelligent Material Systems and Structures. 24(11), 1303-1312(2012)

3. Twiefel, J., Westermann, H.: Survey on broadband techniques for vibration energy harvesting. Journal of Intelligent Material Systems and Structures. 24(11), 1291-1302(2013)

4. He, X.Q., Rafiee, M., Mareishi, S.: Nonlinear dynamics of piezoelectric nanocomposite energy harvesters under parametric resonance. Nonlinear Dynamics. 79(3), 1863-1880(2014)

5. Li, H., Qin, W.: Dynamics and coherence resonance of a laminated piezoelectric beam for energy harvesting. Nonlinear Dynamics. 81(4), 1751-1757(2015)

6. Daqaq, M.F.: Transduction of a bistable inductive generator driven by white and exponentially correlated Gaussian noise. Journal of Sound and Vibration. 330(11), 2554-2564(2011)

7. Daqaq, M.F., Masana, R., Erturk, A., Dane Quinn, D.: On the Role of Nonlinearities in Vibratory Energy Harvesting: A Critical Review and Discussion. Applied Mechanics Reviews. 66(4), 040801(2014)

8. Mitcheson, P.D., Yeatman, E.M., Rao, G.K., Holmes, A.S., Green, T.C.: Energy Harvesting From Human and Machine Motion for Wireless Electronic Devices. Proceedings of the IEEE, (2008)

9. Neiss, S., Goldschmidtboeing, F., Kroener, M., Woias, P.: Analytical model for nonlinear piezoelectric energy harvesting devices. Smart Materials and Structures. 23(10), 105031(2014)

10. Zhang, Y., Tang, L., Liu, K.: Piezoelectric energy harvesting with a nonlinear energy sink. Journal of Intelligent Material Systems and Structures, (2016)

11. Arrieta, A.F., Hagedorn, P., Erturk, A., Inman, D.J.: A piezoelectric bistable plate for nonlinear broadband energy harvesting. Applied Physics Letters. 97(10), 104102(2010)

12. Cottone, F., Vocca, H., Gammaitoni, L.: Nonlinear Energy Harvesting. Physical Review Letters. 102(8)(2009)

13. Erturk, A., Inman, D.J.: Broadband piezoelectric power generation on high-energy orbits of the bistable Duffing oscillator with electromechanical coupling. Journal of Sound and Vibration. 330(10), 2339-2353(2011)

14. Cao, J., Wang, W., Zhou, S., Inman, D.J., Lin, J.: Nonlinear time-varying potential bistable energy harvesting from human motion. Applied Physics Letters. 107(14), 143904(2015)

15. Kim, P., Seok, J.: A multi-stable energy harvester: Dynamic modeling and bifurcation analysis. Journal of Sound and Vibration. 333(21), 5525-5547(2014)

16. Kumar, K.A., Ali, S.F., Arockiarajan, A.: Magneto-elastic oscillator: Modeling and analysis with nonlinear magnetic interaction. Journal of Sound and Vibration. 393, 265-284(2017)

17. Stanton, S.C., McGehee, C.C., Mann, B.P.: Reversible hysteresis for broadband magnetopiezoelastic energy harvesting. Applied Physics Letters. 95(17), 174103(2009)

18. Zhou, S., Cao, J., Lin, J.: Theoretical analysis and experimental verification for improving energy harvesting performance of nonlinear monostable energy harvesters. Nonlinear Dynamics, (2016)

19. Erturk, A., Hoffmann, J., Inman, D.J.: A piezomagnetoelastic structure for broadband vibration energy harvesting. Applied Physics Letters. 94(25), 254102(2009)

20. Daqaq, M.F.: On intentional introduction of stiffness nonlinearities for energy harvesting under white Gaussian excitations. Nonlinear Dynamics. 69(3), 1063-1079(2012) 
21. Zhou, S., Cao, J., Inman, D.J., Lin, J., Liu, S., Wang, Z.: Broadband tristable energy harvester: Modeling and experiment verification. Applied Energy. 133, 33-39(2014)

22. Cao, J.Y., Zhou, S.X., Wang, W., Lin, J.: Influence of potential well depth on nonlinear tristable energy harvesting. Applied Physics Letters. 106(17), 173903(2015)

23. Kim, P., Seok, J.: Dynamic and energetic characteristics of a tri-stable magnetopiezoelastic energy harvester. Mechanism and Machine Theory. 94, 41-63(2015)

24. Kim, P., Son, D., Seok, J.: Triple-well potential with a uniform depth: Advantageous aspects in designing a multi-stable energy harvester. Applied Physics Letters. 108(24), 243902(2016)

25. Panyam, M., Daqaq, M.F.: Characterizing the effective bandwidth of tri-stable energy harvesters. Journal of Sound and Vibration. 386, 336-358(2017)

26. Li, H., Qin, W., Lan, C., Deng, W., Zhou, Z.: Dynamics and coherence resonance of tri-stable energy harvesting system. Smart Materials and Structures. 25(1), 015001(2016)

27. Zhou, Z., Qin, W., Zhu, P.: Improve efficiency of harvesting random energy by snap-through in a quad-stable harvester. Sensors and Actuators A: Physical. 243, 151-158(2016)

28. Zhou, Z., Qin, W., Zhu, P.: A broadband quad-stable energy harvester and its advantages over bi-stable harvester: Simulation and experiment verification. Mechanical Systems and Signal Processing. 84, 158-168(2017)

29. Harne, R.L., Wang, K.W.: Prospects for Nonlinear Energy Harvesting Systems Designed Near the Elastic Stability Limit When Driven by Colored Noise. Journal of Vibration and Acoustics. 136(2), 021009(2013)

30. Halvorsen, E.: Fundamental issues in nonlinear wideband-vibration energy harvesting. Physical Review E. 87(4)(2013)

31. He, Q.F., Daqaq, M.F.: Influence of potential function asymmetries on the performance of nonlinear energy harvesters under white noise. Journal of Sound and Vibration. 333(15), 3479-3489(2014)

32. Erturk, A., Inman, D.J., Piezoelectric energy harvesting. 2011: John Wiley \& Sons.

33. Cao, J., Zhou, S., Inman, D.J., Chen, Y.: Chaos in the fractionally damped broadband piezoelectric energy generator. Nonlinear Dynamics. 80(4), 1705-1719(2015) 